\title{
$K$-TRIVIALITY IN COMPUTABLE METRIC SPACES
}

\author{
ALEXANDER MELNIKOV AND ANDRÉ NIES
}

(Communicated by Julia Knight)

\begin{abstract}
A point $x$ in a computable metric space is called $K$-trivial if for each positive rational $\delta$ there is an approximation $p$ at distance at most $\delta$ from $x$ such that the pair $p, \delta$ is highly compressible in the sense that $K(p, \delta) \leq K(\delta)+O(1)$. We show that this local definition is equivalent to the point having a Cauchy name that is $K$-trivial when viewed as a function from $\mathbb{N}$ to $\mathbb{N}$. We use this to transfer known results on $K$-triviality for functions to the more general setting of metric spaces. For instance, we show that each computable Polish space without isolated points contains an incomputable $K$-trivial point.
\end{abstract}

\section{INTRODUCTION}

Chaitin [6] and Solovay [13] were the first to study $K$-trivial infinite sequences of bits. In the last decade, this notion has turned out to be of key importance for the interactions of computability and randomness.

We identify infinite sequences of bits with subsets of $\mathbb{N}$. Let $K(x)$ denote the prefix-free Kolmogorov complexity of a binary string $x$. This is a variant of the usual plain Kolmogorov complexity $C(x)$ based on a universal machine such that no string in the domain can be an initial segment of another. Recall that a set $A \subseteq \mathbb{N}$ is Martin-Löf random iff $\forall n\left[K\left(A \uparrow_{n}\right) \geq n-O(1)\right]$, namely, the complexity in the sense of $K$ of its initial segments $A \uparrow_{n}$ is close to maximal. By definition, a $K$-trivial set $A$ is far from random: the complexity of its initial segments is minimal up to a constant, namely

$$
K\left(A \uparrow_{n}\right) \leq K(n)+O(1),
$$

where the number $n$ is identified with the string given by its binary expansion. The notion of $C$-triviality is defined in an analogous way. Chaitin [6] showed that each $C$-trivial set is computable and that each $K$-trivial set is $\Delta_{2}^{0}$. Solovay built a $K$-trivial set that is incomputable [13. A much simpler construction of such a set that is also computably enumerable (c.e.) was given in 7]. The coincidence of the $K$-trivial sets with several other classes was shown in 11. For instance, a set $A \subseteq \mathbb{N}$ is called low for $K$ if $K(y) \leq K^{A}(y)+O(1)$ for each string $y$. Nies and Hirschfeldt (see [11]) proved that $K$-triviality is equivalent to lowness for $K$. These and other results show that the $K$-trivial sets are very close to being computable.

Received by the editors October 18, 2011 and, in revised form, October 28, 2011.

2010 Mathematics Subject Classification. Primary 03D32, 03F60.

Key words and phrases. Computable analysis, metric spaces, $K$-triviality.

Both authors were partially supported by the Marsden Fund of New Zealand, grant No. 08UOA-187. 
Many of the questions on the $K$-trivials posed in [10] and elsewhere remain open. For instance, is every $K$-trivial set Turing below an incomplete Martin-Löf random set?1 Does the ideal of $K$-trivial Turing degrees have an exact pair of c.e. degrees?2

1.1. Adapting $K$-triviality to other settings: First steps. We are interested in extending the notion of $K$-triviality to settings more general than subsets of $\mathbb{N}$. First we will look at two minor adaptations: (i) functions from $\mathbb{N}$ to $\mathbb{N}$ and (ii) reals in the unit interval. The first serves as an important tool to understand the general concept of $K$-triviality in computable metric spaces that we will develop later.

(i) Definition (11) can be adapted easily to the case of a function $f$. We encode tuples of natural numbers by binary strings in some effective way. We say $f$ is $K$-trivial if $\forall n\left[K\left(f \uparrow_{n}\right) \leq K(n)+O(1)\right]$. As a consequence of the coincidences in [11], we will show that a function is $K$-trivial if and only if its graph is $K$-trivial as a set.

(ii) One can transfer the notion of $K$-triviality to reals in the unit interval via the binary expansion. We will check that this class of reals is actually independent of the choice of base 2 .

1.2. Computable metric spaces. Our main objective is to develop a theory of $K$-triviality in the general setting of a computable metric space. Recall from [14, 5] that a metric space $(M, d)$ is provided with a computable structure by specifying a dense sequence of points in $M$ such that the pairwise distance is uniformly computable:

Definition 1.1. Let $(M, d)$ be a metric space, and let $\left(\alpha_{i}\right)_{i \in \mathbb{N}}$ be a dense sequence in $M$ without repetitions. We say that $\mathcal{M}=\left(M, d,\left(\alpha_{i}\right)_{i \in \mathbb{N}}\right)$ is a computable metric space if $d\left(\alpha_{i}, \alpha_{k}\right)$ is a computable real uniformly in $i, k$. We call the elements of the sequence $\left(\alpha_{i}\right)_{i \in \mathbb{N}}$ the special points. A point $x \in M$ is called computable if from a positive rational $\delta$ one can compute $p$ such that $d\left(x, \alpha_{p}\right) \leq \delta$.

Example 1.2. Separable metric spaces common in analysis usually have a natural computable structure. For the unit interval $[0,1]$ take the rationals in it. For $\mathbb{R}^{n}$, take $n$-tuples of rationals. For the space of continuous functions $\mathcal{C}[0,1]$ take the polynomials with rational coefficients.

A Cantor space is a metric space where the points are subsets of $\mathbb{N}$ and the distance between two distinct sets is $2^{-k}$ for the least $k$ at which the two sets disagree on membership. A natural computable structure is given by (some effective listing without repetition of) the finite sets. Similarly, Baire space consists of the functions $f: \mathbb{N} \rightarrow \mathbb{N}$. The distance between two distinct functions is $2^{-k}$ for the least $k$ at which their values disagree. The computable structure is given by (some effective listing without repetition of) the functions that have the value 0 for almost all arguments. For each of these examples, the computable points are the objects that are computable in the previously defined sense.

1.3. Adapting $K$-triviality to the general setting of computable metric spaces. We say that a point $x \in M$ is $K$-trivial if for each positive rational $\delta$ there is $p \in \mathbb{N}$ such that

$$
d\left(x, \alpha_{p}\right) \leq \delta \text { and } K(\langle p, \delta\rangle) \leq K(\delta)+O(1) .
$$

\footnotetext{
${ }^{1}$ Added in proof: This has now been solved in the affirmative by combining work of Bienvenu, Greenberg, Kučera, Nies, and Turetsky with work of Day and Miller. See [3].

${ }^{2}$ Added in proof: This has been answered negatively by Barmpalias and Downey.
} 
(Here we fix some effective encoding of the positive rationals by natural numbers and hence binary strings; by $K(\delta)$ we mean the complexity of the string encoding $\delta$.) Note that the pair $(\langle p, \delta\rangle)$ determines an elementary closed ball $\{y: d(y, p) \leq \delta\}$. The intuition is that for each $\delta$, the point $x$ is contained in such a ball that is highly compressible as measured by $\delta$ and $p$. We give fourfold evidence that this is the right generalization of $K$-triviality to a computable metric space $\mathcal{M}$.

1. The definition generalizes previously studied concepts of $K$-triviality.

This class of points coincides with the one defined by (11) above in the case of Cantor space. For the unit interval, definition (2) yields the class of points with a $K$-trivial binary expansion. This establishes that the class is actually independent of the choice of base 2. Barmpalias et al. 1] introduced a notion of $K$-triviality for compact subsets of Cantor space. In Subsection 5.4 we will show that their notion coincides with ours for the metric space of compact sets with Hausdorff distance.

2. Existence result. We show in Theorem 5.7 that every computable complete metric space without isolated points contains a dense set of $K$-trivial incomputable points.

3. In (2) we cannot replace the term $K(\langle p, \delta\rangle)$ by $K(p)$. At first sight one may think that condition (2) should be replaced by

$$
d\left(x, \alpha_{p}\right) \leq \delta \text { and } K(p) \leq K(\delta)+O(1) .
$$

However, this is not the right generalization of $K$-triviality for various reasons. Firstly, we lose the intuition on compressible elementary closed balls mentioned above. Secondly, in the case of Cantor space, the string $A \Gamma_{n}$ in (1) provides a compressible special point $A\left\lceil_{n} 0^{\infty}\right.$ and also the distance of at most $2^{-n}$ away from the point $A$. Thirdly, and most importantly, the weaker condition (3) does not imply usual $K$-triviality in Cantor space: in Proposition 3.7 we build a point $A$ satisfying (3) which is Turing complete, and hence not $K$-trivial.

4. Robustness of the definition. We will see in Corollary 5.5 that $K$-triviality of a point is invariant under the change of computable structure to an equivalent one in the sense of Definition 5.3 below. Furthermore, we will show that in (2) one can restrict $\delta$ to rationals of the form $2^{-n}$ and still obtain the same class of points. Thus (2) can be replaced by an apparently weaker condition: for each $n$ there is $p_{n}$ such that

$$
d\left(x, \alpha_{p_{n}}\right) \leq 2^{-n} \text { and } K\left(\left\langle p_{n}, n\right\rangle\right) \leq K(n)+O(1) .
$$

While this condition is often easier to work with, we refrain from using it in the definition because it seems to depend on the arbitrary choice of distances $2^{-n}$. Unlike the case of Cantor space, these distances play no special role in a general metric space.

1.4. Main result: $K$-trivial points have $K$-trivial Cauchy names. Each $K$ trivial set $A$ satisfies (41) in Cantor space: for each $n$ let $p_{n}$ be the number so that $\alpha_{p_{n}}=A \cap\{0, \ldots, n-1\}$. But $K$-triviality in Cantor space seems to be much stronger than (44): since we can compute the tuple $\left(p_{0}, \ldots, p_{n-1}\right)$ from $A \uparrow_{n}$, we have in fact $K\left(p_{0}, \ldots, p_{n-1}\right) \leq K(n)+O(1)$. In contrast, while (4) says that $\left\langle p_{n}, n\right\rangle$ is compressible for each $n$, it says nothing about the complexity of the whole tuple $\left(p_{0}, \ldots, p_{n-1}\right)$. So it may seem that the "local condition" (44), which talks about each $n$ separately, is inadequate, because $K$-triviality as defined in (11) relies on the 
particular structure of Cantor space. In this case there would be no reasonable way to extend this notion to the general setting of a computable metric space.

Our main result, Theorem 4.2, shows that in fact for each $K$-trivial point we can find a sequence $\left(p_{n}\right)_{n \in \mathbb{N}}$ in (4) such that $K\left(p_{0}, \ldots, p_{n-1}\right) \leq K(n)+O(1)$. Identifying a special point $\alpha_{p}$ with the number $p$, we say that a Cauchy name for a point $x$ is a sequence $\left(p_{s}\right)_{s \in \mathbb{N}}$ of special points converging to $x$ such that $d\left(p_{s}, p_{t}\right) \leq 2^{-s}$ for each $t>s$. The main result states that a point is $K$-trivial if and only if it has a Cauchy name that is $K$-trivial when viewed as a function $\mathbb{N} \rightarrow \mathbb{N}$.

In the proof we build for each constant $b$ a c.e. tree that contains, for each point $x$ that is $K$-trivial via $b$, a path that is $K$-trivial as a function via $2 b+O(1)$ and is a Cauchy name for $x$. To make the tree c.e. we use Solovay functions, studied in 3 .

\section{Preliminaries}

We use the notation in 12. In particular, we write $s \leq^{+} t$ to denote that $s \leq t+O(1)$. Throughout the paper we will use the usual Cantor pairing function $\langle a, b\rangle=a+\frac{1}{2}(a+b)(a+b+1)$ to encode pairs of natural numbers by a single number. We provide some formal definitions of concepts particular to this paper, most of which have already been discussed above.

2.1. $K$-triviality for functions. Fix some effective encoding of tuples $x$ over $\mathbb{N}$ by binary strings so that $K(x)$ is defined for any such tuple. The following extends the definition of $K$-triviality for subsets of $\mathbb{N}$, which can be identified with $\{0,1\}$-valued functions.

Definition 2.1. We say that a function $f: \mathbb{N} \rightarrow \mathbb{N}$ is $K$-trivial if

$$
\exists b \forall n K\left(f \uparrow_{n}\right) \leq K(n)+b .
$$

By the following, $K$-triviality for functions may be reduced to $K$-triviality for sets.

Proposition 2.2. A function $f: \mathbb{N} \rightarrow \mathbb{N}$ is $K$-trivial if and only if its graph $\Gamma=$ $\{\langle n, f(n)\rangle: n \in \mathbb{N}\}$ is $K$-trivial in the usual sense of sets.

Proof. First suppose that $f$ is $K$-trivial. We have

$$
K\left(\Gamma \uparrow_{n(n-1) / 2}\right) \leq^{+} K\left(f \uparrow_{n}\right) \leq^{+} K(n),
$$

since to describe $\Gamma \uparrow_{n(n-1) / 2}$ it is enough to know the values of $f$ up to $n$. For a set $A$, if there is a computable increasing sequence $\left\{q_{n}\right\}_{n \in \omega}$ such that $K\left(A \uparrow_{q_{n}}\right) \leq^{+} K(n)$, then $A$ is $K$-trivial [12, Ex. 5.2.9]. Therefore the set $\Gamma$ is $K$-trivial.

Now suppose that $\Gamma$ is $K$-trivial. Recall that a set $X$ is called low for $K$ if $K(y) \leq^{+} K^{X}(y)$ for each string $y$. By [11], $K$-triviality implies lowness for $K$ (also see [12, Section 5.4]). Thus, $K\left(f \uparrow_{n}\right) \leq^{+} K^{\Gamma}\left(f \uparrow_{n}\right)$. Clearly $K^{\Gamma}\left(f \uparrow_{n}\right) \leq^{+} K(n)$. Thus $K\left(f \uparrow_{n}\right) \leq^{+} K(n)$, as required.

Note that the result does not depend on the particular choice of a pairing function: if we use an alternative pairing function, then the graph of $f$ in terms of that pairing function is $m$-equivalent to $\Gamma$. Hence it is $K$-trivial iff $\Gamma$ is. We could also formulate a similar theorem for other encodings of functions by sets; for instance, a function $f$ is $K$-trivial iff $S$ is $K$-trivial, where $S$ is given by the rule that $1+f(n)$ is the $n+1$-th element of $S$ minus the $n$-th element of $f$. 
Note that the implication from right to left in Proposition 2.2 relies on the hard result of [11, which has a non-uniform proof. It is not known whether Proposition 2.2 is uniform, that is, whether a constant for the $K$-triviality of $f$ can be computed from an index for $\Gamma$ (say, as an $\omega$-c.e. set) and its $K$-triviality constant. We conjecture that this is not the case.

Let $f \oplus g$ be the function $u$ such that $u(2 n)=f(n)$ and $u(2 n+1)=g(n)$. The following is a consequence of the corresponding fact for sets; see [12, 5.2.17].

Corollary 2.3. If $f, g$ are $K$-trivial as functions, then so is $f \oplus g$.

2.2. Solovay functions. Recall that a computable function $h: \mathbb{N} \rightarrow \mathbb{N}$ is called a Solovay function [3] if $\forall r\left[K(r) \leq^{+} h(r)\right]$ and $\exists^{\infty} r\left[K(r)=^{+} h(r)\right]$. (Equivalently, the integer $\liminf _{r}[h(r)-K(r)]$ is finite.) Solovay [13] constructed an example of such a function. The following simpler, recent example is due to Merkle (unpublished). We include the short verification for the sake of completeness.

Fact 2.4. There is a Solovay function $h$.

Proof. Let $\mathbb{U}$ denote the optimal prefix-free machine. Given $r=\langle\sigma, n, t\rangle$, if $t$ is least such that $\mathbb{U}_{t}(\sigma)=n$, define $h(r)=|\sigma|$. Otherwise let $h(r)=r$.

We have $K(r) \leq^{+} h(r)$ because there is a prefix-free machine $M$ which on input $\sigma$ outputs $r=\langle\sigma, \mathbb{U}(\sigma), t\rangle$ if $t$ is least such that $\mathbb{U}_{t}(\sigma)$ halts. If $\sigma$ is also a shortest string such that $\mathbb{U}(\sigma)=n$, then we have $h(r)=|\sigma|=K(n) \leq^{+} K(r)$.

The following generalizes a known fact for sets, also due to Merkle, to the setting of functions.

Fact 2.5. Let $h$ be as in the proof of Fact 2.4. Let $f: \mathbb{N} \rightarrow \mathbb{N}$ be a function such that $\forall r K\left(f \uparrow_{r}\right) \leq h(r)+b$. Then $f$ is $K$-trivial via a constant $b+O(1)$.

Proof. Given $n$, let $\sigma$ be a shortest $\mathbb{U}$-description of $n$, and let $t$ be least such that $\mathbb{U}_{t}(\sigma)=n$. Let $r=\langle\sigma, n, t\rangle$. Then

$$
K\left(f \uparrow_{n}\right) \leq^{+} K\left(f \uparrow_{r}\right) \leq h(r)+b=|\sigma|+b=K(n)+b .
$$

\section{Computable points and $K$-trivial points}

In the following we fix a computable metric space $\mathcal{M}=\left(M, d,\left(\alpha_{i}\right)_{i \in \mathbb{N}}\right)$ as in Definition 1.1. We will use letters $p, q$, etc., for special points in $M$. They will be identified with natural numbers via the listing above. Recall that a point $x$ is computable if one can effectively determine arbitrarily good approximations of $x$ that are special points:

Definition 3.1. We say that $x \in M$ is a computable point if we can effectively in a rational $\delta$ determine $p$ such that $d(x, p) \leq \delta$.

3.1. $K$-trivial points: Definition and examples. A positive rational $\delta$ is viewed as a fraction $\frac{n}{v}$ where $\operatorname{gcd}(n, v)=1$, which we effectively encode by a single natural number $i_{\delta}$. For $i=i_{\delta}$ and $p \in \mathbb{N}$ we let $K(\delta):=K(i)$ and $K(p, \delta):=K(p, i)$. The following is the main definition of the paper. 
Definition 3.2. Let $b \in \mathbb{N}$. We say that a point $x \in M$ is $K$-trivial via $b$, or $K$-trivial $(b)$ for short, if for each positive rational $\delta$ there is a special point $p$ such that

$$
d(x, p) \leq \delta \wedge K(p, \delta) \leq K(\delta)+b .
$$

A point $x \in M$ is called $K$-trivial if it is $K$-trivial via some $b$.

Choosing a different effective encoding of the rationals will merely lead to a different constant $b$ in (5), without affecting the set of $K$-trivial points. Thus we might as well use the canonical encoding of a positive rational $\delta$ : If $\delta=\frac{n}{v}$ where $\operatorname{gcd}(n, v)=1$, let $i_{\delta}=\langle n, v\rangle$. (This yields the listing $1,1 / 2,2,1 / 3,3,1 / 4,2 / 3, \ldots$ of the positive rationals.)

Clearly each computable point is $K$-trivial. In some cases there are no others:

Example 3.3. There is an infinite compact computable metric space $\mathcal{M}$ with an incomputable point such that each $K$-trivial point is computable.

Proof. Let $\Omega$ denote Chaitin's halting probability. We have $\Omega=\lim _{s} \Omega_{s}$, where $\Omega_{s}=\sum\left\{2^{-|\sigma|}: \mathbb{U}_{s}(\sigma) \downarrow\right\}$. Let $\mathcal{M}$ be the computable metric space with domain $\left\{\Omega_{s}: s \in \mathbb{N}\right\} \cup\{\Omega\}$, the metric inherited from the unit interval and with the computable structure given by $\alpha_{s}=\Omega_{s}$.

Assume for a contradiction that $\Omega$ is a $K$-trivial point. Given $n$, pick $p=\Omega_{s}$ as in (5) for $\delta=2^{-n}$. We can compute $\Omega_{s} \uparrow_{n}$ (the first $n$ bits of the binary expansion of $\left.\Omega_{s}\right)$ from $p$ and $n$. Since $d\left(\Omega, \Omega_{s}\right) \leq 2^{-n}$, this shows that $K\left(\Omega \uparrow_{n}\right)$ $\leq K(p, n)+O(1) \leq K(n)+O(1)$, which contradicts $K\left(\Omega \uparrow_{n}\right) \geq n-O(1)$ for sufficiently large $n$.

In Theorem 5.7 we will show that each computable complete metric space $\mathcal{M}$ without isolated points contains an incomputable $K$-trivial point. Instead of giving a direct construction, we will derive this from the corresponding fact in Cantor space using two facts: Proposition 5.4 below, stating that $K$-triviality is preserved by a computable map from one metric space to another, and a result of Brattka and Gherardi stating that there is a 1-1 computable map from Cantor space into $\mathcal{M}$, which hence also preserves incomputability of points.

3.2. Number and distribution of $K$-trivial points for a constant $b$. Firstly, we note that few numbers $p$ can satisfy the second inequality in (5):

Fact 3.4. For $\delta \in \mathbb{Q}^{+}$, we have $|\{p \in \mathbb{N}: K(p, \delta) \leq K(\delta)+b\}|=O\left(2^{b}\right)$.

Proof. Chaitin [6] proved that there are only $O\left(2^{b}\right)$ strings $v$ of length $n$ such that $K(v) \leq K(n)+b$. To prove the fact at hand, one adapts, for instance, the proof in $[12,2.2 .26]$ of his result, with the change that one lets $M(\sigma)=n$ if $\mathbb{U}(\sigma)=\langle i, n\rangle$, where $n \in \mathbb{N}$ encodes $\delta$.

We now show that a similar bound holds for the number of points that are $K$-trivial(b). Furthermore, such a point $x$ is determined by a highly compressible special point $p$ close by.

Proposition 3.5. Let $b \in \mathbb{N}$. (i) At most $O\left(2^{b}\right)$ many $x \in M$ are $K$-trivial $(b)$. (ii) There is a rational $\delta>0$ as follows. If a point $x$ is $K$-trivial via $b$, then there is a special point $p$ with $K(p, \delta) \leq K(\delta)+b$ such that $x$ is the only $K$-trivial $(b)$ point with $d(x, p) \leq \delta$. 
Proof. Suppose that distinct points $x_{1}, \ldots, x_{k} \in M$ are $K$-trivial $(b)$. Pick a rational $\delta>0$ such that $2 \delta$ is less than $d\left(x_{i}, x_{j}\right)$ for any $i \neq j$, and choose $p_{i}$ for $x_{i}, \delta$ according to (5). Then all the $p_{i}$ are distinct. By Fact 3.4 this implies that $k=O\left(2^{b}\right)$, hence (i) holds. If $k$ is chosen maximal, then $x_{i}$ is the only $K$-trivial $(b)$ point $x$ such that $d\left(x, p_{i}\right) \leq \delta$, which establishes (ii).

3.3. Dyadic $K$-triviality. We mostly work with the apparently weaker form of Definition 3.2 of $K$-triviality in metric spaces, where $\delta$ only ranges over rationals of the form $2^{-n}$. This was discussed in (4) of the introduction.

Definition 3.6. A point $x \in M$ is dyadically $K$-trivial via $b$ if for each $n \in \mathbb{N}$ there is a special point $p$ such that

$$
d(x, p) \leq 2^{-n} \wedge K(p, n) \leq K(n)+b .
$$

Clearly, a point that is $K$-trivial via $v$ is dyadically $K$-trivial via $v+O(1)$. We will show in our main result, Theorem 4.3 that being dyadically $K$-trivial via $b$ already implies having a Cauchy name that is $K$-trivial as a function via $2 b+O(1)$, which in turn easily implies being $K$-trivial via $2 b+O(1)$. Thus, up to a computable change in constants, dyadic $K$-triviality is the same as $K$-triviality.

For the metric spaces in Example 1.2 we already have a notion of $K$-triviality. We now verify that this coincides with dyadic $K$-triviality.

Suppose a point $f$ in Cantor or Baire space is $K$-trivial in the usual sense. Then it is clearly dyadically $K$-trivial: given $n$, let $p$ be the special point determined by $f \uparrow_{n}$, namely $p(i)=f(i)$ for $i<n$ and $p(i)=0$ for $i \geq n$. The $p$ is a witness in (6). Conversely, each dyadically $K$-trivial point $f$ is $K$-trivial in the usual sense, because given $n$, if $p$ is a witness in (6), we have $K\left(f \uparrow_{n}\right) \leq K(p, n)+O(1)$.

Recall that the unit interval $[0,1]$ is a computable metric space with the usual distance function and the computable structure given by some effective listing without repetition of the rationals in $[0,1]$. Suppose a point $x$ has binary expansion $0 . A$, where $A$ is an infinite bit sequence (set). If $A$ is $K$-trivial, then as a witness $p$ in (6) we may use the dyadic rational $0 .\left(A \uparrow_{n}\right)$. Conversely, suppose $x \in[0,1]$ is dyadically $K$-trival. Given $n$ let $p$ be a witness in (6), and let $\sigma$ be the first $n$ bits in the binary expansion of $p$. Then $K(\sigma) \leq K(p, n)+O(1)$; furthermore, $0 . \sigma-0 .\left(A \uparrow_{n}\right)=c 2^{-n}$ for some $c \in\{-1,0,1\}$. Therefore $K\left(A \uparrow_{n}\right) \leq K(n)+O(1)$.

3.4. We cannot replace the term $K(p, \delta)$ in Definition 3.2 by $K(p)$. We provide the example showing that, even in Cantor space, (3) is not an adequate generalization of $K$-triviality. The "dyadic" version of (3) is that

$$
\forall n \exists p\left[d(x, p) \leq 2^{-n} \wedge K(p) \leq K(n)+O(1)\right] .
$$

This is in fact equivalent to (3), because from $\delta>0$ one can compute the least $n$ such that $2^{-n} \leq \delta$. Then a witness $p$ for $n$ in (7) is also a witness for $\delta$ in (3). Recall from [7] that a $K$-trivial is not Turing complete.

Proposition 3.7. There is a Turing complete $\Pi_{1}^{0}$ set $A \in\{0,1\}^{\mathbb{N}}$ satisfying condition (7).

Proof. For a string $\alpha$ over $\{0,1\}$, let $g(\alpha)$ be the longest prefix of $\alpha$ that ends in 1 , and $g(\alpha)=\emptyset$ if there is no such prefix. We say that a set $A$ is weakly $K$-trivial if

$$
\forall n\left[K\left(g\left(A \uparrow_{n}\right)\right) \leq^{+} K(n)\right] .
$$


This implies (7): given $n$ let $p=g\left(A \uparrow_{n}\right) 0^{\infty}$; then $d(p, A) \leq 2^{-n}$. (As an aside, we note that every $K$-trivial set is weakly $K$-trivial. Every weakly $K$-trivial set with an infinite computable subset is already $K$-trivial by [12, Ex. 5.2.9].)

We now build a Turing complete $\Pi_{1}^{0}$ set $A$ that is weakly $K$-trivial. We maintain the condition that

$$
\forall i \forall w\left[\gamma_{i}<w \rightarrow K(w)>i\right]
$$

where $\gamma_{i}$ is the $i$-th element of $A$. This implies that $A$ is Turing complete, as follows. We build a prefix-free machine $N$. When $i$ enters $\emptyset^{\prime}$ at stage $s$, we declare that $N\left(0^{i} 1\right)=s$. This implies $K(s) \leq i+d$ for some fixed coding constant $d$. Now $i \in \emptyset^{\prime} \leftrightarrow i \in \emptyset_{\gamma_{i+d}}^{\prime}$, which implies $\emptyset^{\prime} \leq_{\mathrm{T}} A$.

We let $A=\bigcap A_{s}$, where $A_{s}$ is a cofinite set effectively computed from $s, A_{0}=\mathbb{N}$, $[s, \infty) \subseteq A_{s}$, and $A_{s+1} \subseteq A_{s}$ for each $s$. We view $\gamma_{i}$ as a movable marker; $\gamma_{i}^{s}$ denotes its position at stage $s$, which is the $i$-th element of $A_{s}$.

Construction of $A$ and a prefix-free machine $M$.

Stage 0. Let $A_{0}=\mathbb{N}$.

Stage $s>0$. Suppose that there is $w$ such that $i:=K_{s}(w)<K_{s-1}(w)$. By convention, we may assume that $w$ is unique and $w<s$. Thus, there is a new computation $\mathbb{U}_{s}(\sigma)=w$ with $|\sigma|=i$ at stage $s$.

If $w \leq \gamma_{i}^{s-1}$, then let $A_{s}=A_{s-1}$. If $w>\gamma_{i}^{s-1}$, then, to maintain (8) at stage $s$, we move the marker $\gamma_{i}$ : we let $A_{s}=A_{s-1}-\left[\gamma_{i}^{s-1}, s\right)$, which results in $\gamma_{i+k}^{s}=s+k$ for $k \geq 0$, while $\gamma_{j}^{s}=\gamma_{j}^{s-1}$ for $j<i$.

In any case, declare $M(\sigma)=g\left(A_{s} \uparrow_{w}\right)$.

Verification. Clearly, each marker $\gamma_{i}$ moves at most $2^{i+1}$ times, so $A=\bigcap_{s} A_{s}$ is an infinite co-c.e. set. Furthermore, condition (8) holds because it is maintained at each stage of the construction.

We show by induction on $s$ that

$$
\forall n\left[K\left(g\left(A_{s} \uparrow_{n}\right)\right) \leq^{+} K_{s}(n)\right]
$$

For $s=0$ the condition is vacuous. Now suppose $s>0$ and (9) holds for $s-1$. We may suppose that $w$ as in stage $s$ of the construction exists; otherwise (9) holds at stage $s$ by inductive hypothesis.

As in the construction, let $i=K_{s}(w)$ and let $\sigma$ be the string of length $i$ such that $\mathbb{U}_{s}(\sigma)=w$. If $w \leq \gamma_{i}^{s}$, then $A_{s}=A_{s-1}$, so setting $M(\sigma)=g\left(A_{s} \uparrow_{w}\right)$ maintains (91).

Now suppose that $w>\gamma_{i}^{s}$. Let $n<s$. We verify (9) at stage $s$ for $n$.

If $n \leq \gamma_{i}^{s-1}$, then $A_{s} \Upsilon_{n}=A_{s-1} \Upsilon_{n}$ and $K_{s}(n)=K_{s-1}(n)$, so the condition holds at stage $s$ for $n$ by inductive hypothesis. Now suppose that $n>\gamma_{i}^{s-1}$. By (8) at stage $s-1$ we have $K_{s-1}(n)>i$, and hence $K_{s}(n) \geq i$ (equality holds if $n=w)$. Because $n, w>\gamma_{i}^{s-1}$ and we move the marker $\gamma_{i}$ at stage $s$, we have $g\left(A_{s} \Upsilon_{n}\right)=g\left(A_{s} \uparrow_{w}\right)$. Thus, setting $M(\sigma)=g\left(A_{s} \Upsilon_{w}\right)$ ensures that condition (9) holds at stage $s$ for $n$. 


\section{A point is $K$-trivial iff it has a $K$-trivial Cauchy name}

As before we fix a computable metric space $\mathcal{M}=\left(M, d,\left(\alpha_{i}\right)_{i \in \mathbb{N}}\right)$.

Definition 4.1. A sequence $\left(p_{s}\right)_{s \in \mathbb{N}}$ of special points is called a Cauchy name if $d\left(p_{s}, p_{t}\right) \leq 2^{-s}$ for each $s, t \in \mathbb{N}, s<t$. If $x=\lim _{s} p_{s}$, we say that $\left(p_{s}\right)_{s \in \mathbb{N}}$ is a Cauchy name for $x$. Note that $d\left(x, p_{s}\right) \leq 2^{-s}$ for each $s$.

Via the underlying listing of special points $\left(\alpha_{i}\right)_{i \in \mathbb{N}}$ we may view a Cauchy name as a function $\mathbb{N} \rightarrow \mathbb{N}$. It is clear that a point is computable in the sense of Definition 3.1 if and only if it has a computable Cauchy name. Indeed the latter is often taken as the definition of computable points.

Our main result is the corresponding fact for $K$-triviality.

Theorem 4.2. Let $x$ be a point in a computable metric space $\mathcal{M}$.

(i) If $x$ has a Cauchy name that is $K$-trivial as a function via $u \in \mathbb{N}$, then $x$ is a $K$-trivial point via $u+O(1)$.

(ii) If $x$ is a $K$-trivial point via $v \in \mathbb{N}$, then $x$ has a Cauchy name that is $K$-trivial as a function via $2 v+O(1)$.

We begin with proving the easier part (i). Recall that before Definition 3.2 we fixed an effective encoding of positive rationals $\delta$ by natural numbers $i_{\delta}$. It is easy to verify that $\delta \geq 2^{-i_{\delta}}$ for each $\delta$.

Suppose now that the function $f$ is $K$-trivial via $u$ and is a Cauchy name for $x$. Given $\delta>0$ let $i=i_{\delta}$. If $n$ is least such that $\delta \geq 2^{-n}$, then we can take $p=f(n)$ as a witness for $K$-triviality in the sense of (5): we have $i \geq n$, and hence

$$
K(f(n), i) \leq K\left(f \uparrow_{i+1}\right)+O(1) \leq K(i)+u+O(1) .
$$

Since $K(i)$ is the same as $K(\delta)$ and $K(p, i)$ is the same as $K(p, \delta)$, this shows (i).

In Definition 3.6 we defined the auxiliary concept of dyadic $K$-triviality and noted that being $K$-trivial via $v$ implies being dyadically $K$-trivial via $v+O(1)$. The following lemma will close the circle by establishing (ii).

Lemma 4.3. Suppose that the point $x \in M$ is dyadically $K$-trivial via $b$. Then $x$ has a Cauchy name $f$ that is $K$-trivial as a function via $2 b+O(1)$.

Proof. After adding a natural number to the Solovay function $h$ from Fact 2.4, we may suppose that $\forall r K(r) \leq h(r)$.

The c.e. tree $T$. By a tree we mean a set $T \subseteq \mathbb{N}^{<\omega}$ that is closed under taking prefixes. As usual, $[T]$ denotes the set of (infinite) paths of a tree $T$. We define a c.e. tree $T$ such that each $f \in[T]$ is a Cauchy name after leaving out $f(0)$ and $f(1)$. Let $T=\bigcup_{s} T_{s}$, where we define the trees

$$
\begin{aligned}
T_{s}=\left\{\left(p_{1}, \ldots, p_{v}\right):\right. & \forall i \leq v\left[K_{s}\left(p_{i}, i\right) \leq h(i)+b\right] \\
& \left.\wedge \forall i<v\left[d\left(p_{i}, p_{i+1}\right) \leq 2^{-i+1}\right]\right\} .
\end{aligned}
$$

Example 4.4. The following computable metric space $\mathcal{M}$ may serve as an illustration for what follows. Let the special points of $\mathcal{M}$ be all the pairs $\langle r, n\rangle$, where $r \in\{0,1\}$ and $n \in \mathbb{N}$. We declare

$$
d(\langle 0, n\rangle,\langle 1, n\rangle)=2^{-n} \text { and } d(\langle r, n),\langle k, n+1\rangle)=2^{-n-1} .
$$


All the special points are isolated. There is only one non-isolated point, namely $x=\lim _{n}\langle 0, n\rangle$. This point is computable. Each sequence $\left(p_{n}\right)_{n \in \mathbb{N}}$ of the form $\left(\left\langle r_{n}, n\right\rangle\right)_{n \in \mathbb{N}}$ is a Cauchy name for $x$. For an appropriate $b$, (마) holds for each $n$ via $p_{n}$.

A thin c.e. subtree $G$ of $T$. In the setting of Example4.4, $T$ is a full 2-branching tree, consisting of all the tuples of the form $\left(\left\langle r_{0}, 0\right\rangle,\left\langle r_{1}, 1\right\rangle, \ldots,\left\langle r_{v}, v\right\rangle\right)$ where $r_{i} \in\{0,1\}$. This shows that $T$ may contain lots of Cauchy names consisting of witnesses for dyadic $K$-triviality; we cannot expect that each such Cauchy name is $K$-trivial as a function. Therefore we will prune $T$ to a c.e. subtree $G$ that is so thin that all strings $\tau$ in it are compressible in the sense that $K(\tau) \leq h(|\tau|)+b+O(1)$. Hence each infinite path is dyadically $K$-trivial by Fact 2.5 .

We say that a special point $p \in \mathbb{N}$ is present at level $n$ of a tree $B \subseteq \mathbb{N}<\omega$ if there is $\eta \in B$ such that $\eta$ has length $n$ and ends in $p$. While $G$ is only a thin subtree of $T$, we will ensure that each special point present at level $n$ of $T$ is also present at level $n$ of $G$. This will show that $[G]$ still contains a function that (after leaving out the first two values) is a Cauchy name for $x$. (In Example 4.4 there are only two labels at each level of $T$, so for $G \subseteq T$ we can simply take the tuples where each $r_{i}$ is 0 , except possibly the last. Then the only path of $G$ is a computable Cauchy name of the limit point $x$.)

We will build a computable enumeration $\left(G_{s}\right)_{s \in \mathbb{N}}$ of the tree $G$ where $G_{s}$ is a tree contained in $T_{s}$ for each $s$.

Why each string in $G$ can be compressed. Suppose that at a certain stage, a new leaf labelled $p$ appears at level $n$ of $T$ but is not yet present at level $n$ of $G$. Suppose also that $p$ is a successor on $T$ of a node labelled $q$. Inductively, $q$ is already present at level $n-1$ of $G$; that is, there already is a node $\bar{\eta}$ of length $n-1$ on $G$ that ends in $q$. Since $p$ is present at level $n$ of $T$, there is a $\mathbb{U}$-description showing that $K(p, n) \leq h(n)+b$ (that is, there is a string $w$ with $|w| \leq h(n)+b$ such that $\mathbb{U}(w)=\langle p, n\rangle)$. Since $p$ is not present at level $n$ of $G$, this $\mathbb{U}$-description is "unused". Hence we can use it as a description of a new node $\eta=\bar{\eta}\urcorner$ in $G$. This ensures that $K(\eta) \leq h(n)+b+O(1)$.

Note that we make use of the fact that once a string $\eta$ in $G$ is compressible at a stage, it remains so at all later stages. This is the reason we need the Solovay function $h$. If we tried to satisfy the condition $K(\eta) \leq K(n)+b+O(1)$, we might fail, because $K(n)$ on the right side could decrease later on. We also needed the Solovay function to ensure that $T$ is c.e.

In the formal construction, we build a prefix-free machine $L$ (see [12, Chapter 2]) to give short descriptions of these nodes. The argument above is implemented via maintaining conditions (12) and (13) below.

A slower computable enumeration $\left(\widetilde{T}_{s}\right)_{s \in \mathbb{N}}$ of $T$. We define a computable enumeration $\left(\widetilde{T}_{s}\right)_{s \in \mathbb{N}}$ of $T$ that grows "one leaf at a time". The $\widetilde{T}_{s}$ are subtrees of $T_{s}$. Let $\widetilde{T}_{0}$ consist only of the empty string. If $s>0$ and $\widetilde{T}_{s-1}$ has been defined, see whether there is $\tau \in T_{s}-\widetilde{T}_{s-1}$. If so, choose $\tau$ least in some effective numbering of $\mathbb{N}^{<\omega}$. Pick $v$ maximal such that $\tau \uparrow_{v} \in \widetilde{T}_{s-1}$, and put $\tau \uparrow_{v+1}$ into $\widetilde{T}_{s}$. Clearly we have $\widetilde{T}_{s} \subseteq T_{s}$ and $T=\bigcup_{s} \widetilde{T}_{s}$.

Three conditions that need to be maintained at each stage. For strings $\tau, \eta \in \mathbb{N}<\omega$ we write $\tau \sim \eta$ if they have the same length and end in the same element. Recall 
that each label present at a level $n$ of $T$ needs also to be present at level $n$ of $G$. Actually, in the construction we ensure that for each stage $s$, each label $p$ that is present at a level $n$ of $\widetilde{T}_{s}$ is also present at level $n$ of $G_{s}$ :

$$
\forall \tau \in \widetilde{T}_{s} \exists \eta \in G_{s}[\tau \sim \eta] .
$$

To make sure that each $\eta \in G$ satisfies $K(\eta) \leq h(|\eta|)+b+O(1)$, we construct, along with $\left(G_{s}\right)_{s \in \mathbb{N}}$, a computable enumeration $\left(L_{s}\right)_{s \in \mathbb{N}}$ of (the graph of) a prefixfree machine $L$. Let $m, n$ range over natural numbers and $v, w$ over strings. We maintain at each stage $s$ the conditions

$$
\begin{aligned}
& \forall \eta \in G_{s} \forall m\left[0<m \leq|\eta| \rightarrow \exists v\left[|v| \leq h(m)+b \wedge L_{s}(v)=\eta \uparrow_{m}\right]\right] ; \\
& \text { if } \mathbb{U}_{s}(w)=\langle p, n\rangle, \text { then }\left[w \in \operatorname{dom}\left(L_{s}\right) \rightarrow p \text { is at level } n \text { of } G_{s}\right] .
\end{aligned}
$$

Construction of $\left(G_{s}\right)_{s \in \mathbb{N}}$ and $\left(L_{s}\right)_{s \in \mathbb{N}}$.

Stage 0 . Let $G_{0}$ contain only the empty string. Let $L_{0}=\emptyset$. Clearly the conditions (11), 12, 13) hold for $s=0$.

Stage $s>0$. Inductively we assume that (11), (12), (13)) hold for $s-1$.

If $\widetilde{T}_{s}-\widetilde{T}_{s-1}$ is empty, go to the next stage. Otherwise there is a unique $\tau \in$ $\widetilde{T}_{s}-\widetilde{T}_{s-1}$. Let $n=|\tau|$. By the definition of the computable enumeration $\left(\widetilde{T}_{r}\right)_{r \in \mathbb{N}}$, we have $\tau=\bar{\tau}^{\wedge} p$ for some $\bar{\tau} \in \widetilde{T}_{s-1}$. Since $\tau \in T_{s}$, by the definition in (10) we have $\mathbb{U}_{s}(w)=\langle p, n\rangle$ for some $\mathbb{U}$-description $w$ such that $|w| \leq h(n)+b$.

If $p$ is already present at level $n$ of $G_{s-1}$, then go to the next stage. Otherwise, by (13) for $s-1$, we have $w \notin \operatorname{dom}\left(L_{s-1}\right)$; i.e., we have not yet used $w$ as an $L$-description.

By (11) for $s-1$, there is $\bar{\eta} \in G_{s-1}$ such that $\bar{\tau} \sim \bar{\eta}$. Now let $\eta=\bar{\eta}^{\wedge} p$. (Note that $\eta \in T_{s}$ because $\bar{\eta} \in G_{s-1} \subseteq T_{s}$ and $K_{s}(p, n) \leq h(n)+b$.) Put $\eta$ into $G_{s}$. Set $L_{s}(w)=\eta$. Then conditions ((11), (12), (13) ) hold at stage $s$. Go to the next stage.

Verification. Given a function $f: \mathbb{N} \rightarrow \mathbb{N}$, let $\widehat{f}$ denote the function given by $\widehat{f}(n)=$ $f(n+2)$. If $f \in[T]$, then $\widehat{f}$ is a Cauchy name.

Note that the prefix-free machine $L=L_{b}$ is obtained uniformly in $b$, so we can build a prefix-free machine $M$ such that $M\left(0^{b} 1 \sigma\right)=L_{b}(\sigma)$ for each binary string $\sigma$. Hence, if $f \in[G]$, then by condition (12) and Fact 2.5, $\widehat{f}$ is $K$-trivial as a function via $2 b+O(1)$. The following now concludes the proof of the lemma.

Claim 4.5. There is $f \in[G]$ such that $\widehat{f}$ is a Cauchy name of $x$.

Since $x$ is dyadically $K$-trivial, for each $n$ we can choose $p_{n}$ as in (6). Then $\left(p_{1}, \ldots, p_{n}\right) \in T$. So by (11) we can choose a string $\eta_{n} \in G$ of length $n$ that ends in $p_{n}$.

For each $n$ there are only finitely many $p$ such that $K(p, n) \leq h(n)+b$. So each level of $T$ is finite. Thus, by König's Lemma, there is an infinite path $f$ on the subtree of $G$ consisting of the strings that are a prefix of some $\eta_{n}$. For each $r>0$ there is $n$ such that $f \uparrow_{r} \preceq \eta_{n}$. So $n \geq r$. Hence

$$
d(f(r-1), x) \leq d\left(f(r-1), p_{n}\right)+d\left(p_{n}, x\right) \leq 2^{-r+2}+2^{-n} \leq 2^{-r+3} .
$$

This shows that $\widehat{f}$ is a Cauchy name for $x$.

This concludes Lemma 4.3 and thereby establishes Theorem 4.2 
4.1. An analog of Theorem 4.2 for plain Kolmogorov complexity $C$. We adapt the foregoing proof. We say that a function $f: \mathbb{N} \rightarrow \mathbb{N}$ is $C$-trivial via $u$ if $C\left(f \uparrow_{n}\right) \leq C(n)+u$ for each $n$.

A point $x \in M$ is called $C$-trivial via $v$ if for each positive rational $\delta$ there is a special point $p$ such that

$$
d(x, p) \leq \delta \wedge C(p, \delta) \leq C(\delta)+v .
$$

Clearly (i) of Theorem 4.2 holds in the setting of plain complexity $C$, via the same proof. To prove an analog of (ii) for $C$, we replace the Solovay function $h$ by a logarithm function. Let $\log _{2} n$ denote the largest integer $k$ such that $2^{k} \leq n$. Clearly, with a shift in constants, condition (14) implies its dyadic variant: for each $n$ there is a special point $p$ such that $d(x, p) \leq 2^{-n} \wedge C(p, n) \leq C(n)+v+O(1)$. Since $C(n) \leq{ }^{+} \log _{2} n$, this in turn implies the condition

$$
\forall n \exists p\left[d(x, p) \leq 2^{-n} \wedge C(p, n) \leq \log _{2} n+b\right]
$$

(where $b=v+O(1)$ ).

We now obtain an analog of Lemma 4.3

Proposition 4.6. Suppose that (15) holds for a point $x$. Then $x$ has a Cauchy name $f$ such that $C\left(f \uparrow_{n}\right) \leq \log _{2} n+2 b+O(1)$.

Proof. Let $h(n)=\log _{2} n+d$, where the constant $d$ is chosen so that $h(n) \geq C(n)$ for each $n$. Now we follow the proof of Theorem 4.2(ii) with this definition of $h$. The machines $L_{b}$ and $M$ are defined as before but are based on a plain universal machine. So they now become plain machines.

This leads to a characterization of computable points via algorithmic information theory.

Corollary 4.7. A point $x$ is $C$-trivial iff it is computable.

Proof. The implication from right to left is clear. For the converse, adapting the proof of Chaitin's result in [12, Thm. 5.2.20(ii)] to functions shows that each function $f$ as in the foregoing proposition is computable. So $x$ has a computable Cauchy name.

\section{Preservation Results and existence of $K$-trivial points}

We apply our main result, Theorem 4.2, in order to obtain information on $K$ trivial points that is not at all obvious from Definition 3.2 First we provide some more background on computable metric spaces.

\subsection{Computable maps and equivalent computable structures.}

Definition 5.1. Let $\mathcal{M}, \mathcal{N}$ be computable metric spaces. A map $F: \subseteq M \rightarrow N$ is called computable if there is a Turing functional $\Phi$ such that for each $x$ in the domain of $F$ and for every Cauchy name $\alpha$ for $x, \Phi^{\alpha}$ is a Cauchy name for $F(x)$.

Recall that a computable map $F$ is called effectively uniformly continuous if there is a computable function $h: \mathbb{N} \rightarrow \mathbb{N}$ such that for $x, y \in M, d(x, y) \leq 2^{-h(n)}$ implies $d(F(x), F(y)) \leq 2^{-n}$. (For instance, if $F$ is Lipschitz with constant $2^{c}$, then $F$ is effectively uniformly continuous via $h(n)=n+c$.) We now show that for such $F$, the Turing functional $\Phi$ above can be chosen to be a weak truth-table reduction; that is, the use is computably bounded. 
Proposition 5.2. Suppose a computable map $F$ is effectively uniformly continuous via $h$. Then there is a Turing functional $\Phi$ as in Definition 5.1 such that for each $\alpha, n$, the use of $\Phi^{\alpha}(n)$ is bounded by $h(n+2)+1$.

Proof. Suppose $F$ is computable via the Turing functional $\widetilde{\Phi}$. To define the functional $\Phi$, given a Cauchy name $\alpha$ for a point $x$, let $\alpha[n]$ denote the Cauchy name $\gamma$ that follows $\alpha$ up to $h(n+2)$ and then repeats; that is, $\gamma(i)=\alpha(i)$ for $i<h(n+2)$ and $\gamma(i)=\alpha(h(n+2))$ for $i \geq h(n+2)$. Let

$$
\Phi^{\alpha}(n)=\widetilde{\Phi}^{\alpha[n]}(n+2) .
$$

Then $h(n+2)+1$ is a use bound as required. To show that $\beta=\Phi^{\alpha}$ is a Cauchy name for $y=F(x)$, note that $d\left(x, \alpha_{h(n+2)}\right) \leq 2^{-h(n+2)}$, and hence $d\left(y, F\left(\alpha_{h(n+2)}\right)\right) \leq$ $2^{-(n+2)}$. Furthermore, since $\alpha[n]$ is a Cauchy name for $\alpha(h(n+2))$, we have $d\left(F\left(\alpha_{h(n+2)}\right), \beta_{n}\right) \leq 2^{-(n+2)}$. Therefore

$$
d\left(y, \beta_{n}\right) \leq d\left(y, F\left(\alpha_{h(n+2)}\right)\right)+d\left(F\left(\alpha_{h(n+2)}\right), \beta_{n}\right) \leq 2^{-(n+1)} .
$$

Therefore $d\left(\beta_{s}, \beta_{t}\right) \leq 2^{-s}$ for $t \geq s$, and $\beta$ converges to $y$.

Definition 5.3. We say that computable structures $\left(q_{i}\right)_{i \in \mathbb{N}}$ and $\left(r_{i}\right)_{i \in \mathbb{N}}$ on a metric space $(M, d)$ are equivalent if the identity and its inverse are computable when the identity is viewed as a map $\mathcal{M} \rightarrow \mathcal{N}$, where $\mathcal{M}=\left(M, d,\left(q_{i}\right)_{i \in \mathbb{N}}\right)$ and $\mathcal{N}=$ $\left(M, d,\left(r_{i}\right)_{i \in \mathbb{N}}\right)$.

For instance, if we apply a computable permutation to the special points, we obtain an equivalent structure. In particular, up to equivalence the examples in subsection 1.2 are independent of the particular effective listings of the special points.

5.2. Preservation of $K$-triviality. The following generalizes the fact [1] that the class of $K$-trivial sets is closed downward under Turing reducibility.

Proposition 5.4. Let $\mathcal{M}, \mathcal{N}$ be computable metric spaces, and let the map $F: M \rightarrow$ $N$ be computable. If $x$ is $K$-trivial in $\mathcal{M}$, then $F(x)$ is $K$-trivial in $\mathcal{N}$.

Proof. Let $\alpha$ be a $K$-trivial Cauchy name for $x$. Since $F$ is computable, there is a Cauchy name $\beta \leq_{T} \alpha$ for $F(x)$. Then $\beta$ is $K$-trivial by Proposition [2.2, and the result of [11] that $K$-triviality for sets is closed downward under $\leq_{\mathrm{T}}$.

If $F$ is effectively uniformly continuous, then one can also give a direct proof which avoids the hard result from [11. Moreover, from a $K$-triviality constant for $x$ one can effectively obtain a $K$-triviality constant for $F(x)$, which is not true in the general case.

To see this, note that by Proposition 5.2 we have $\beta=\Phi^{\alpha}$ for a Turing functional $\Phi$ with use bounded by some computable function $g$. Then

$$
K\left(\beta \uparrow_{n}\right) \leq^{+} K\left(\alpha \uparrow_{g(n)}\right) \leq^{+} K(g(n)) \leq^{+} K(n) .
$$

The increase in constants is fixed, because it only depends on $\Phi$ and $g$.

Since the identity map is Lipschitz, we obtain that $K$-triviality in a computable metric space is invariant under changing of the computable structure to an equivalent one: 
Corollary 5.5. Suppose a point $x$ is $K$-trivial via $b$ with respect to a computable structure on a metric space. Then for any equivalent structure on the same space, $x$ is $K$-trivial via $b+O(1)$.

The first author has shown that all computable structures on the unit interval are equivalent [9]. This means that $K$-triviality is intrinsic to the unit interval as a metric space.

5.3. Existence of non-computable $K$-trivial points. A Polish (i.e., complete separable metric) space is said to be perfect if it has no isolated points. In the following we take Cantor space $\{0,1\}^{\mathbb{N}}$ as the computable metric space with the usual computable structure of Example 1.2

Proposition 5.6 (4, Prop. 6.2]). Suppose the computable Polish space $\mathcal{M}$ is perfect. Then there is a computable injective map $F:\{0,1\}^{\mathbb{N}} \rightarrow \mathcal{M}$ which is Lipschitz with constant 1 .

Theorem 5.7. Let $\mathcal{M}$ be a perfect computable Polish space. Then for every special point $p \in M$ and every $\delta>0$ there exists a non-computable $K$-trivial point $x$ such that $d(x, p) \leq \delta$.

Proof. We may assume $\delta \in \mathbb{Q}$. Note that $\{x: d(x, p) \leq \delta\}$ with the inherited computable structure is again a perfect computable metric space $\mathcal{N}$. By the result of Brattka and Gherardi there is a computable injective Lipschitz map $F:\{0,1\}^{\mathbb{N}} \rightarrow \mathcal{N}$.

Let $A$ be a non-computable $K$-trivial point in Cantor space. Then $x=F(A)$ is $K$-trivial in $\mathcal{N}$, and hence in $\mathcal{M}$, by Proposition 5.4, actually only the easier case of Lipschitz functions discussed after Proposition 5.4 is needed.

As Brattka and Gherardi point out before Proposition 6.2, the inverse of $F$ is computable (on its domain). Thus, if $x$ is computable, then so is $A$, which is not the case.

5.4. $K$-trivial compact sets. Given a Polish space $M$, let $\mathbb{K}(M)$ denote the Polish space of compact subsets of $M$ with the Hausdorff distance (the maximum distance that a point in one set can have from the other set). If $M$ is a computable metric space, then $\mathbb{K}(M)$ carries a natural computable structure where the special points are the finite sets of special points in $M$. Thus we have a notion of $K$-trivial compact sets.

If $M$ is Cantor space $\{0,1\}^{\mathbb{N}}$, then the computable structure on $\mathbb{K}(M)$ is easily seen to be equivalent in the sense of Definition 5.3 to the one where the special points are the clopen sets. Barmpalias et al. [1 studied the notion of $K$-triviality for compact subsets $V$ in Cantor space. For instance, they built a non-empty $K$-trivial $\Pi_{1}^{0}$ class $V$ without computable paths. We will show that their notion coincides with ours.

They noted that the definition they gave originally is equivalent to requiring that the subtree $T_{V}=\{\sigma:[\sigma] \cap V \neq \emptyset\}$ of $\{0,1\}<\omega$ is $K$-trivial. For the latter condition it suffices to ask that $K\left(T_{V}[n]\right) \leq^{+} K(n)$ for each $n$, where $T_{V}[n]$ is (a suitable string encoding of) the finite set $\left\{\sigma \in T_{V}:|\sigma|=n\right\}$.

Fact 5.8. Let $V \subseteq\{0,1\}^{\mathbb{N}}$ be closed. Then $V$ is a $K$-trivial point in $\mathbb{K}\left(\{0,1\}^{\mathbb{N}}\right) \Leftrightarrow$ $T_{V}$ is $K$-trivial as a set. 
Proof. $\Leftarrow$ : Let $P_{n}=\bigcup\left\{[\sigma]: \sigma \in T_{V}[n]\right\}$. Then the $P_{n}$ are witnesses for dyadic $K$-triviality of $V$ according to Definition 3.6 .

$\Rightarrow$ : For each $n$ let $P_{n}$ be a clopen set such that $d\left(P_{n}, V\right)<2^{-n}$ and $K\left(P_{n}, n\right) \leq^{+}$ $K(n)$. Then for each string $\sigma$ of length $n$ we have $[\sigma] \cap V \neq \emptyset \leftrightarrow[\sigma] \subseteq P_{n}$. This shows that $T_{V}$ is $K$-trivial.

\section{REFERENCES}

[1] G. Barmpalias, D. Cenzer, J. B. Remmel, and R. Weber. $K$-triviality of closed sets and continuous functions. J. Logic Comput., 19(1):3-16, 2009. MR2475639 (2009k:03072)

[2] L. Bienvenu, A. Day, N. Greenberg, A. Kučera, J. S. Miller, A. Nies, and D. Turetsky. Computing $K$-trivial sets by incomplete random sets. Preprint, 2012.

[3] L. Bienvenu and R. Downey. Kolmogorov complexity and Solovay functions. In Symposium on Theoretical Aspects of Computer Science (STACS 2009), LIPIcs. Leibniz Int. Proc. Inform., 3, Schloss Dagstuhl. Leipniz-Zent. Inform., Wadern, 2009, pages 147-158. MR2870648

[4] V. Brattka and G. Gherardi. Borel complexity of topological operations on computable metric spaces. J. Logic Comput., 19(1):45-76, 2009. MR2475641 (2010g:03099)

[5] V. Brattka, P. Hertling, and K. Weihrauch. A tutorial on computable analysis. In S. Barry Cooper, Benedikt Löwe, and Andrea Sorbi, editors, New Computational Paradigms: Changing Conceptions of What Is Computable, pages 425-491. Springer, New York, 2008. MR2762094(2012e:03142)

[6] G. Chaitin. Information-theoretical characterizations of recursive infinite strings. Theoretical Computer Science, 2:45-48, 1976. MR0413595 (54:1709)

[7] R. Downey, D. Hirschfeldt, A. Nies, and F. Stephan. Trivial reals. In Proceedings of the 7th and 8th Asian Logic Conferences, pages 103-131, Singapore University Press, Singapore, 2003. MR2051976 (2005a:03089)

[8] D. Loveland. A variant of the Kolmogorov concept of complexity. Information and Control, 15:510-526, 1969. MR0274200 (42:9075)

[9] A. Melnikov. Computability and Structure. Ph.D. thesis, University of Auckland, 2012.

[10] J. Miller and A. Nies. Randomness and computability: Open questions. Bull. Symbolic Logic, 12(3):390-410, 2006. MR2248590 (2007c:03059)

[11] A. Nies. Lowness properties and randomness. Adv. in Math., 197:274-305, 2005. MR2166184 (2006j:68052)

[12] A. Nies. Computability and Randomness, volume 51 of Oxford Logic Guides. Oxford University Press, Oxford, 2009. MR2548883(2011i:03003)

[13] R. Solovay. Handwritten manuscript related to Chaitin's work. IBM Thomas J. Watson Research Center, Yorktown Heights, NY, 215 pages, 1975.

[14] K. Weihrauch. Computable Analysis. Springer, Berlin, 2000. MR1795407 (2002b:03129)

Department of Computer Science, University of Auckland, Private Bag 92019, AuckLAND, NEW ZEALAND

Current address: Department of Mathematics, Nanyang Technological University, 50 Nanyang Avenue, Singapore 639798

Department of Computer Science, University of Auckland, Private Bag 92019, AuckLAND, NEW ZEALAND

E-mail address: andre@cs.auckland.ac.nz 